This item was submitted to Loughborough's Research Repository by the author.

Items in Figshare are protected by copyright, with all rights reserved, unless otherwise indicated.

\title{
Atomistic insights of multiple stacking faults in CdTe thin-film photovoltaics: A DFT study
}

PLEASE CITE THE PUBLISHED VERSION

https://doi.org/10.1109/PVSC.2018.8547934

PUBLISHER

(C) IEEE

VERSION

AM (Accepted Manuscript)

LICENCE

CC BY-NC-ND 4.0

\section{REPOSITORY RECORD}

Watts, Michael J., Stephen R. Yeandel, Roger Smith, Michael Walls, and Pooja M. Panchmatia. 2019. "Atomistic Insights of Multiple Stacking Faults in Cdte Thin-film Photovoltaics: A DFT Study". figshare. https://hdl.handle.net/2134/36320. 


\title{
Atomistic Insights of Multiple Stacking Faults in CdTe Thin-Film Photovoltaics: A DFT Study
}

\author{
Michael J. Watts, ${ }^{*}$ Stephen R. Yeandel, ${ }^{*}$ Roger Smith, ${ }^{*}$ J. Michael Walls ${ }^{\dagger}$ and Pooja M. Panchmatia* \\ *Loughborough University, Loughborough, Leicestershire, LE11 3TU, United Kingdom \\ ${ }^{\dagger}$ Centre for Renewable Energy Systems Technology, Loughborough University, Leicestershire, LE11 3TU, \\ United Kingdom
}

\begin{abstract}
Stacking faults in CdTe were studied using DFT simulations. Twin and tetrahedral stacking fault energies are significantly lower than previously suggested, strongly correlating with their high density observed experimentally. No long range ordering was found for tetrahedral stacking faults while a resistance for polytype clustering was calculated. All experimentally observed faults were shown to be electronically benign when considered in isolation but increased density may produce shallow electron trap states.

Index Terms-CdTe, stacking faults, density functional theory, thin films, photovoltaics.
\end{abstract}

\section{INTRODUCTION}

Polycrystalline CdTe thinfilm solar cells exhibit high efficiency and have comparatively low manufacturing costs making them the dominant chalcogenide for solar technology. A post-deposition $\mathrm{CdCl}_{2}$ annealing treatment is required to raise the efficiency from $<1 \%$ to viable levels, with a record research cell efficiency of $>20 \%$ [1]. However, the mechanism for this performance enhancement is not well understood.

As-deposited CdTe films contain very high densities of stacking faults, with $48 \%$ of layers deviating from the normal ABC stacking [2]. This is much reduced post chloride treatment. Abbas et al. [3] found further annealing removes the chlorine, reduces efficiency and causes stacking faults to return. The atomic-scale complexity of cells after chlorine treatment means experimental methods have difficulty separating the effects of possible improvement mechanisms so computational simulations have been employed to understand this further.

Yoo et al. [4] used Density Functional Theory (DFT) to investigate the energetics and electronic properties of various stacking fault structures in bulk CdTe. They report tetrahedraltype faults and $\Sigma 3(111)$ twins to be electronically benign, and suggest polytype faults are detrimental. All stacking faults show low stacking fault energies, in agreement with Yan et al. [5], explaining their high density. Twins were found to be the most stable, suggesting why they alone are able to persist through chlorine treatment [4]. Polytype faults are significantly less favourable than other faults and are therefore not likely to form in significant numbers, with Fiducia et al. [2] finding no experimental evidence of polytype faults.
Yan et al. [6] observed the so-called 'buried wurtzite' phase in CdTe films. This consists of a small area of wurtzite ABAB stacking in the otherwise ABC stacking of CdTe's zinc blende stucture. As the bulk wurzite phase of CdTe has a wider bandgap than zinc blende, with a conduction band offset of $+65 \mathrm{meV}$, they theorised that this may have a detrimental effect on cell performance. However, this assumes that the small wurtzite regions within zinc blende CdTe have the same properties as pure bulk wurtzite, which may not necessarily be the case for buried wurtzite in-situ.

To date, modelling studies [4], [5] have been focused on isolated faults and whilst this provides useful insights, these models are unable to capture the effects of multiple faults seen experimentally. Therefore in this work, a systematic modelling study of multiple stacking faults and their effects on the electronic structure, and hence photovoltaic performance, have been investigated. Furthermore, since stacking fault energies are small, accurate calculations, using larger structures than those previously studied, are warranted.

\section{Methodology}

DFT calculations were performed using the Vienna $A b$ initio Simulation Package (VASP). [7] Structures and stacking fault energies were calculated using the PBEsol exchangecorrelation functional [8] which predicts a bulk lattice constant of $6.50 \AA$, in close agreement with the experimental value of $6.48 \AA$ [9]. Structures were relaxed until HellmanFeynman forces on all atoms converged to $<0.01 \mathrm{eV} / \AA$. Single point calculations with a hybrid functional incorporating $25 \%$ Hartree-Fock exact exchange mixing were then performed on optimized structures for accurate electronic properties. Projector augmented wave (PAW) pseudopotentials and a plane wave electronic basis set are used for all calculations.

Stacking faults were added to a clean (111) structure of 12 CdTe formula units in height, shown in Figure 1 a, with resultant fault structures shown in Figures 1p,c and 2 Buried wurtzite was simulated using a structure of $50 \%$ wurtzite and $50 \%$ zincblende phases. Both the twin and buried wurtzite cells contain two fault interfaces to mantain periodicity and therefore their stacking fault energies are quoted per fault to remain consistent with the lone faults. Intrinsic stacking faults 
are created by removing a layer from the stacking sequence, e.g. $\mathrm{ABC} * \mathrm{BCABC}$ while extrinsic faults involves adding an extra layer, e.g. $\mathrm{ABCB} A B C A B C$. This means directly comparing clean and fault-containing structures would require large structures with multiple faults which are prohibitively expensive for hybrid DFT calculations. The stacking fault energies are therefore calculated using a scaled number of clean layers using equation 1 in which $\mathrm{E}_{\mathrm{Fault}}$ is the total energy of the stacking fault structure, $\mathrm{E}_{\text {clean }}$ is the energy of one CdTe formula unit, $\mathrm{n}$ is the number of formula units in the stacking fault structure, A is the cross sectional area of the cell in the plane of the stacking fault and $x$ is the number of stacking fault interfaces required to maintain cell periodicity.

$$
\text { Stacking Fault Energy }=\frac{1}{x A}\left(E_{\text {fault }}-n E_{\text {clean }}\right)
$$

Yoo et al.'s [4] results are well reproduced using equivalent simulation parameters and atomic structures [4], [11]. However, when testing with an increased k-point grid and basis set cut off energy, to improve accuracy, the stacking fault energies do not converge, although the bulk unit cell remains adequately converged. When calculating such small deviations in the stacking fault energies, which are also inherently small, the convergence of the calculations is paramount. Therefore, an increased plane wave basis set cutoff energy of $500 \mathrm{eV}$ and $\Gamma$-centered Monkhorst Pack kpoint grid of $8 \times 8 \times 1$ for (111) orientated cells were used with larger orthorhombic structures. The orthorhombic structures are compared to equivalent hexagonal structures as used by Yoo et al. [4], [11] in Figure 3.

Yoo et al.'s [4] hybrid functional band-gap results also correspond with the use of HSEsol [12] rather than HSE06 [13] as reported. [4] The most recent pseudopotentials available in VASP produce poor agreement with experiment using HSEsol, predicting a band-gap of $1.39 \mathrm{eV}$ for the bulk unit cell, so HSE06 was used instead in this work which produces a band-gap of $1.53 \mathrm{eV}$ in better agreement with the experimental value of $1.48 \mathrm{eV}$.[4] For the HSE06 calculations (which as explained can be very computationally expensive) the smaller hexagonal structures, a reduced cut off of $300 \mathrm{eV}$ and an increased k-point grid of $10 \times 10 \times 1$ were used to maintain a similar convergence. Tests with larger orthorhombic cells produced negligible deviations from these values. Band offsets were calculated by aligning core states in fault-containing cells with those of the clean (111) orientated structure.

\section{RESULTS AND DISCUSSION}

Table II lists the stacking fault energies for each stacking fault type calculated by Yoo et al. [4], [11] compared to this work. It is clear that our calculated values, with higher accuracy, for both tetrahedral and twin faults are significantly lower than those calculated previously [4], [5]. However, the new lower values are in much better agreement with the experimental literature value of $9 \mathrm{~mJ} / \mathrm{m}^{2}$. [14] Having said this, the trend is still representative, where the twins are more (a)

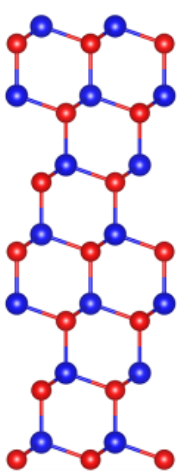

(b)

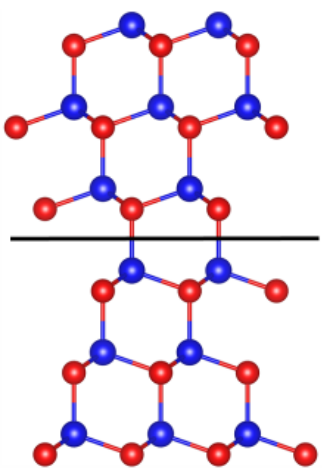

(c)

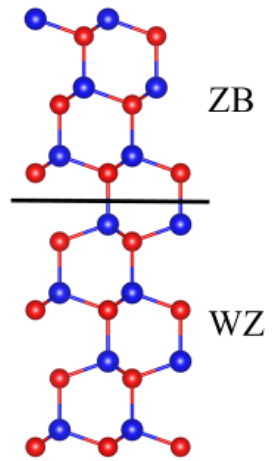

Fig. 1. Structures of the (a) clean, (b) twin and (c) buried wurtzite phase structures. KEY: blue $=\mathrm{Cd}$, red $=\mathrm{Te}$

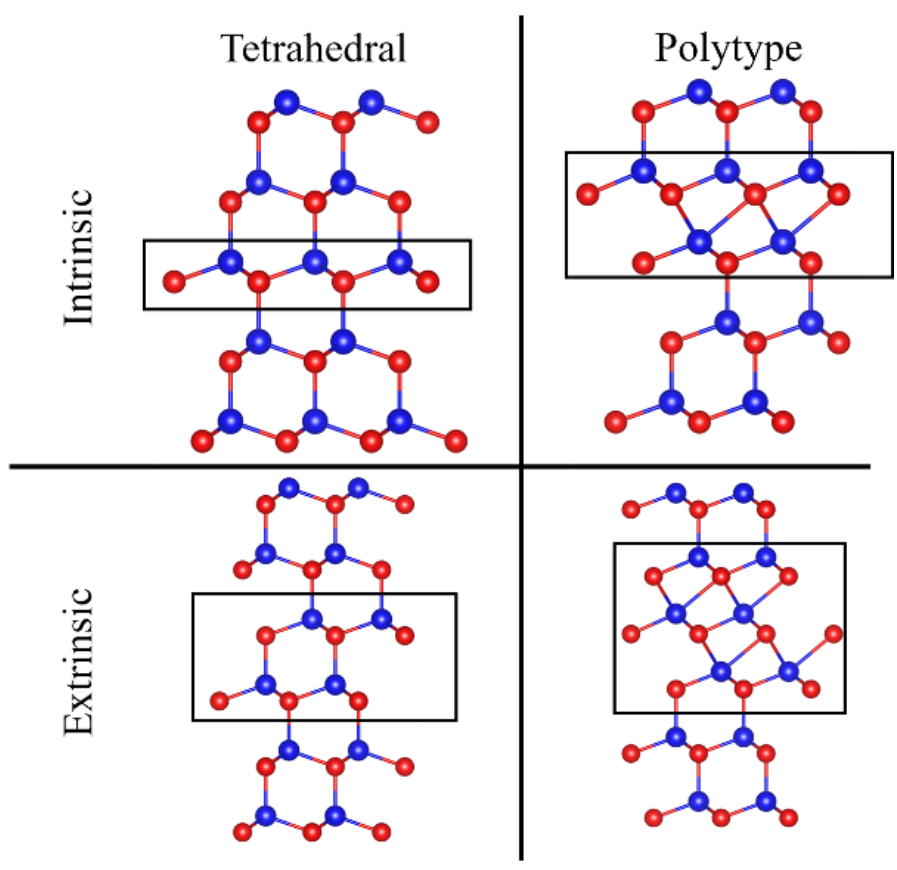

Fig. 2. Schematic representation of the various intrinsic and extrinsic stacking fault structures studied. KEY: blue $=\mathrm{Cd}$, red $=\mathrm{Te}$

stable than the tetrahedral faults, with the difference now only $6.6 \mathrm{~mJ} / \mathrm{mm}^{2}$. Such a small difference casts doubt on simple relative stability arguments for twins persisting through $\mathrm{Cl}$ treatment and warrants further work to establish a mechanism that could explain why the tetrahedral faults are selectively removed upon chlorine treatment.

The polytype faults are calculated to have approximately double the stacking fault energy as previously reported [4]. Our extrinsic polytype fault is calculated to be almost double the stacking fault energy of the intrinsic polytype. This is more in line with what one would expect, as the extrinsic structures could be considered as two intrinsic faults clustered 
(a)

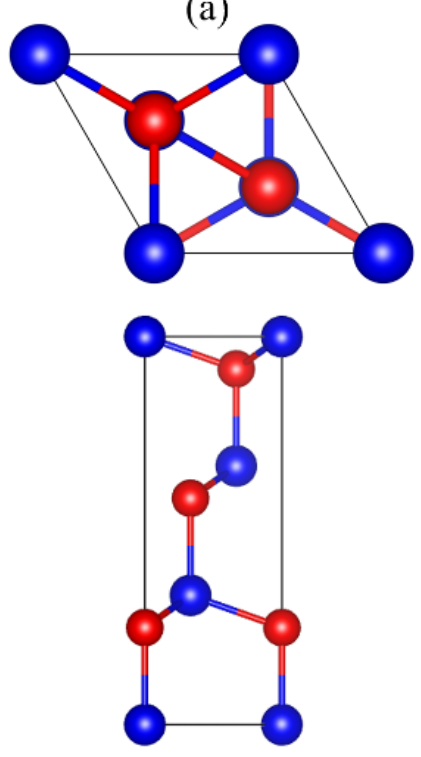

(b)

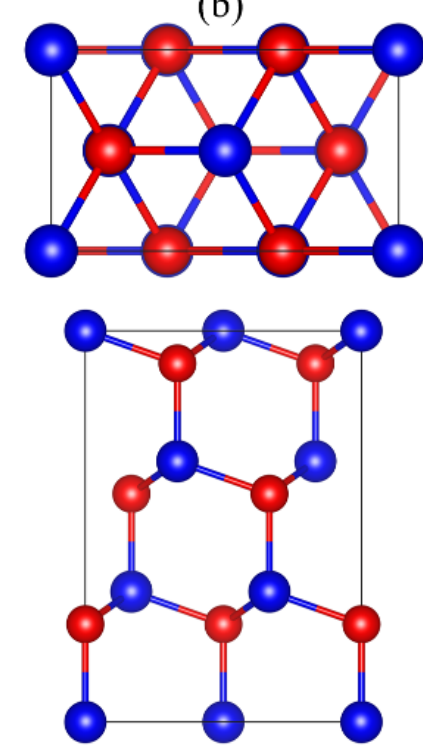

Fig. 3. Comparison of the (a) hexagonal, as used by Yoo et al., [4] and (b) orthorhombic cell structures used for stacking fault energies in this work. KEY: blue $=\mathrm{Cd}$, red $=\mathrm{Te}$

TABLE I

STACKING FAULT ENERGIES

\begin{tabular}{|l|l|l|l|}
\hline \multirow{2}{*}{ Structure } & \multicolumn{3}{|c|}{ Stacking Fault Energy $\left(\mathrm{mJ} / \mathrm{m}^{2}\right)$} \\
\cline { 3 - 4 } & Ref [4], [1] & Current Work \\
\hline \multirow{2}{*}{ Tetrahedral } & Intrinsic & 26.7 & 12.4 \\
\cline { 2 - 4 } & Extrinsic & 32.1 & 11.6 \\
\hline \multirow{2}{*}{ Polytype } & Intrinsic & 219.5 & 401.4 \\
\cline { 2 - 4 } & Extrinsic & 234.6 & 780.2 \\
\hline \multicolumn{2}{|l|}{ Twin } & 16.2 & 5.8 \\
\hline \multicolumn{2}{|l|}{ Buried wurtzite } & & 18.2 \\
\hline
\end{tabular}

together, and suggests a resistance for polytype fault clustering. However, the same trend is not seen for tetrahedral faults, in which the extrinsic is most stable. The twin, which may be considered a cluster of many tetrahedral faults, is also more stable than either such fault in isolation.

The interaction between faults has been studied by varying the number of clean layers between the faults. Combinations of intrinsic-intrinsic, extrinsic-extrinsic and intrinsic-extrinsic tetrahedral faults are shown in Figure 4 The first points in this figure (labelled zero) corresponds to two faults adjacent to each other. It should be noted that the stacking fault energy per fault is equivalent to a twin when the faults are adjacent and to individual tetrahedral faults when separated. From Figure 4 we see that separation does not affect the stacking fault energies, suggesting that there is no long range order between multiple tetrahedral faults. This also implies that random diffusion is the primary mechanism for faults to cluster together into large twins. This suggests that the faults can be fully removed and do not coalesce to form twins on chlorine treatment unless the action of chlorine affects fault clustering dynamics.

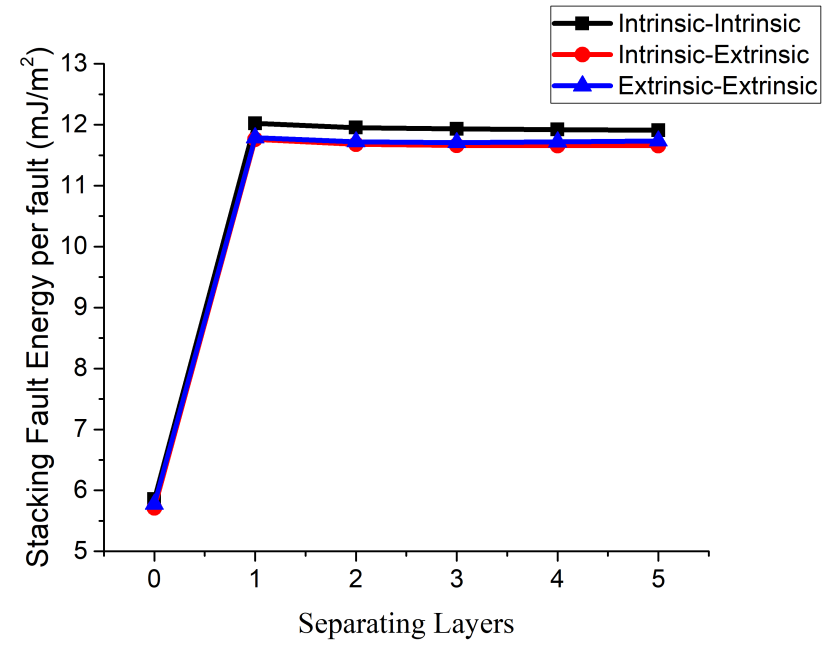

Fig. 4. Stacking fault energies of two tetrahedral stacking faults with varying separation layers

A similar investigation into the clustering behaviour of polytype faults reveals that only lone faults are stable, with any separation between the two faults causing a reconstruction to bulk-like structures. Although energetically unfavourable, polytype stacking faults can be expected to form in small numbers, however the tendency to reconstruct explains why there is a complete lack of experimental observation.

The effect of stacking faults on the band-gap is an important factor to consider since experimental groups have alluded to the removal of stacking faults to the increase in efficiency after $\mathrm{Cl}$ treatment[2] - [5]. In order to accurately calculate the band gaps, computationally expensive nontrivial hybrid functionals combined with relatively high convergence factors are required. The effects of stacking faults on the CdTe bandgap are listed in Table $\Pi$ and indicate that twins, tetrahedral faults and the buried WZ structures do not significantly deviate from the bulk band-gap. This suggests that the assumption that the buried wurtzite phase behaves as bulk wurtzite [6] is flawed. Polytype faults exhibit significant reduction of the bandgap, moving CdTe away from the peak of the ShockleyQueisser limit [15], and suggest potential trap states above the valence band maximum which would have a detrimental effect on the performance. However, polytype faults are not observed experimentally and therefore do not explain the reduced efficiency of the CdTe cells before $\mathrm{Cl}$ treatment [2].

When considering both band-gap and band offsets, no experimentally observed stacking fault is predicted to be detrimental to cell performance when considered in isolation. However, the band offsets of the structure containing two intrinsic stacking faults separated by 4 clean CdTe layers are potentially damaging by creating an electron trap state $60 \mathrm{meV}$ below the conduction band. 
TABLE II

BANDGAPS AND BAND OFFSETS

\begin{tabular}{|l|l|l|l|l|}
\hline \multicolumn{2}{|l|}{ Structure } & Bandgap (eV) & VBM (eV) & CBM (eV) \\
\hline Clean & 1.53 & & \\
\hline \multirow{2}{*}{ Tetrahedral } & Intrinsic & 1.55 & -0.01 & 0.01 \\
\cline { 2 - 5 } & Extrinsic & 1.54 & -0.04 & -0.03 \\
\hline \multirow{2}{*}{ Polytype } & Intrinsic & 1.44 & 0.07 & -0.02 \\
\cline { 2 - 5 } & Extrinsic & 1.37 & 0.09 & -0.07 \\
\hline Twin & 1.55 & -0.04 & -0.02 \\
\hline 2x Intrinsic tetrahedral & 1.54 & -0.08 & -0.06 \\
\hline Buried WZ & 1.55 & -0.02 & 0.00 \\
\hline
\end{tabular}

\section{CONCLUSION}

In this work, DFT calculations using accurate convergence parameters have been performed for a variety of isolated and clustered stacking faults. The stacking fault energy for tetrahedral stacking faults is significantly reduced when simulating a larger orthorhombic cell compared to the previously reported hexagonal cell [4]. Intrinsic and extrinsic tetrahedral faults have very similar stacking fault energies and exhibit no long range interactions as the stacking fault energies remain unchanged once faults are separated. Polytype faults are significantly higher in energy than tetrahedral faults and exhibit resistance to clustering but no overall long range interactions are seen. High densities of polytype stacking faults reconstruct into bulk structures explaining their lack of experimental observation [2].

Twins, lone tetrahedral faults and the buried wurtzite phase are shown to be electronically benign while polytype faults cause significant reduction from the clean band-gap value with potential trap states above the valence band maximum. The observed buried wurtzite phase is benign. However, increased density of separated intrinsic tetrahedral stacking faults may cause carrier transport difficulties. More work is therefore needed to establish the mechanism of removal of the experimentally seen high density stacking faults the whether this contributes to the improved cell performance after $\mathrm{Cl}$ treatment seen experimentally.

\section{ACKNOWLEDGMENT}

The Authors would like to acknowledge the fruitful collaboration with Tom Fiducia at the Centre for Renewable Energy and Sustainable Technology (CREST) in Loughborough and useful discussions with Prof. Aron Walsh at Imperial College London. This paper recognises the use of the "Hydra" High Performance System at Loughborough University. Via our membership of the UK's HPC Midlands+ Consortium, which is funded by EPSRC (EP/P020232/1), this work used the Loughborough HPC resource, ATHENA. Furthermore, the authors would like to acknowledge the help of the Science-IT team at Loughborough. Additional funding was provided by Royal Society of Chemistry's Solid State Chemistry Group.

\section{REFERENCES}

[1] M.A. Green, Y. Hishikawa, W. Warta, E.D. Dunlop, D.H. Levi, J. Hohl-Ebinger, A.W.H. Ho-Baillie,"Solar cell efficiency tables (version 50), Progress in Photovoltaics, 25, 7, pp 668676, 2017

[2] T. Fiducia, A. Abbas, K. Barth, W. Sampath and M. Walls,'Intragranular Defects in As-Deposited and Cadmium Chloride-Treated Polycrystalline Cadmium Telluride Solar Cells in 43rd IEEE Photovoltaics Specialist Conference, p. 3366-3390, 2016

[3] A.Abbas, D. Swanson, A. Munshi, K.L. Barth, W.S. Sampath, G.D. West, J.W. Bowers, P.M. Kaminski and J.M. Walls,"The effect of a post-activation annealing treatment on thin film cdte device performance in 42nd IEEE Photovoltaics Specialist Conference, p.1-6, 2015

[4] S. Yoo, K. Butler, A. Soon, A. Abbas, J. Walls and A. Walsh, "Identification of critical stacking faults in thin-film CdTe solar cells Applied Physics Letters, 105, 062104, 2014.

[5] Y.Yan and M. M. Al-Jassim, "Energetics and effects of planar defects in CdTe, Journal of Applied Physics, 90, 3952, 2001

[6] Y. Yan, M.M. Al-Jassim, K.M. Jones, S.-H. Wei and S.B. Zhang, "Observation and first-principles calculation of buried wurtzite phases in zinc-blende CdTe thin films, Applied Physics Letters, vol. 77, p.1461-1463, 2000

[7] G. Kresse and J. Furthmuller, "Efficient iterative schemes for ab initio total-energy calculations using a plane-wave basis set", Physical Review B, vol. 54, no. 16, p. 11169-11186, 1996

[8] J.P.. Perdew, A. Ruzsinszky, G.I.Csonka, O.A. Vydrov, G.E. Scuseria, L.A.. Constantin, X. Zhou and K. Burke, "Restoring the Density-Gradient Expansion for Exchange in Solids and Surfaces", Physical Review Letters, 100, 136406, 2008

[9] M. K. Rabadanov, I. A. Verin, Y. M. Ivanov, and V. I. Simonov, "Refinement of the atomic structure of CdTe single crystals," Crystallography Reports, vol. 46, no. 4, pp. 636-641, 2001.

[10] G. Kresse, and J. Joubert, "From ultrasoft pseudopotentials to the projector augmented wave method", Physical Review B, 59, 1758 (1999).

[11] A.Walsh, Imperial College London, Personal Communication, 3rd Jan 2017

[12] L. Schimka, J. Harl and G. Kresse, "Improved hybrid fuctional for solids: The HSEsol functional", The Journal of Chemical Physics, 134, 024116, 2011

[13] J.Heyd, G.E. Scuseria and M. Ernzerhof, "Hybrid functionals based in a screened Coulomb potential", The Journal of Chemical Physics, 118, 8207, 2003

[14] S.Takeuchi and K. Suzuki, "Stacking Fault Energies of Tetrahedrally Coordinated Crystals", Physica Status Solidi (a), 171, 99,1999

[15] W. Shockley and H.J. Queisser, "Detailed Balance Limit of Efficiency of p-n Junction Solar Cells Journal of Applied Physics, 32, 510, 1961 